University of Nebraska - Lincoln

DigitalCommons@University of Nebraska - Lincoln

3-23-1998

\title{
Evolution of a Plasma Waveguide Created during Relativistic- Ponderomotive Self-Channeling of an Intense Laser Pulse
}

Shouyuan Chen

University of Michigan, Ann Arbor, schen6@unl.edu

\section{G.S. Sarkisov}

University of Michigan, Ann Arbor

Anatoly Maksimchuk

University of Michigan, tolya@umich.edu

R. Wagner

University of Michigan, Ann Arbor

Donald P. Umstadter

University of Nebraska-Lincoln, donald.umstadter@unl.edu

Follow this and additional works at: https://digitalcommons.unl.edu/physicsumstadter

Part of the Physics Commons

Chen, Shouyuan; Sarkisov, G.S.; Maksimchuk, Anatoly; Wagner, R.; and Umstadter, Donald P., "Evolution of a Plasma Waveguide Created during Relativistic-Ponderomotive Self-Channeling of an Intense Laser Pulse" (1998). Donald Umstadter Publications. 26.

https://digitalcommons.unl.edu/physicsumstadter/26

This Article is brought to you for free and open access by the Research Papers in Physics and Astronomy at DigitalCommons@University of Nebraska - Lincoln. It has been accepted for inclusion in Donald Umstadter Publications by an authorized administrator of DigitalCommons@University of Nebraska - Lincoln. 


\title{
Evolution of a Plasma Waveguide Created during Relativistic-Ponderomotive Self-Channeling of an Intense Laser Pulse
}

\author{
S.-Y. Chen, G. S. Sarkisov, A. Maksimchuk, R. Wagner, and D. Umstadter \\ Center for Ultrafast Optical Science, University of Michigan, Ann Arbor, Michigan 48109
}

(Received 10 October 1997)

\begin{abstract}
An on-axis plasma density depression channel was observed during and after the passage of a relativistically and ponderomotively self-guided laser pulse through a plasma. Optical interferometry was used to produce time-resolved plasma density distributions, revealing the formation of a plasma waveguide. These results were complemented by the guiding of a collinear trailing pulse. [S0031-9007(98)05623-3]

PACS numbers: 52.75.Di, 52.35.Mw, 52.40.Nk
\end{abstract}

There is currently much interest in the interaction of high-intensity ultrashort laser pulses with plasmas, both from the standpoint of basic physics and potential applications in advanced fusion energy, x-ray lasers, and ultrahigh-gradient electron accelerators [1-3]. The success of these applications depends critically on longdistance propagation of laser pulses at relativistic intensities $\left(\sim 10^{18} \mathrm{~W} / \mathrm{cm}^{2}\right.$ for $1-\mu \mathrm{m}$-wavelength light). In order to reach such high intensities, laser pulses are usually focused tightly, which, due to diffraction, results in a short interaction length $\left(\sim 1-2\right.$ Rayleigh ranges, $\left.Z_{R}=\pi r_{0}^{2} / \lambda\right)$. Several methods have been proposed to extend the propagation distance of pulses beyond this diffraction limit, as reviewed in [4].

For optical guiding of laser pulses in plasmas, the radial profile of the index of refraction, $n(r)$, must have a maximum on axis, causing the wavefront to curve inward and the laser beam to converge. When this focusing force is strong enough to counteract the diffraction of the beam, the laser pulse can propagate over a long distance and maintain a small cross section (laser channel). The index of refraction for a plasma is given by $n(r)=1-$ $\left(\omega_{p}^{2} / \omega_{0}^{2}\right)\left[n_{e}(r) / n_{e 0} \gamma(r)\right]$, where $\omega_{p}$ is the plasma frequency for electron density $n_{e 0}, \omega_{0}$ is the laser frequency, $n_{e}(r)$ is the radial distribution of electron density, and $\gamma(r)$ is the relativistic factor associated with the electron motion transverse to the laser propagation. The factor $\gamma$ depends on the normalized vector potential, $a_{0}$, by $\gamma=\sqrt{1+a_{0}^{2}}$, where $a_{0}=\gamma v_{0 s} / c=e E / m_{e} \omega c=$ $8.5 \times 10^{-10} \lambda[\mu \mathrm{m}] I^{1 / 2}\left[\mathrm{~W} / \mathrm{cm}^{2}\right]$. It can readily be seen from this that an on-axis maximum of $n(r)$ can be created through modification of the radial profile of $\gamma$ and/or $n_{e}$.

When a laser beam with an intensity profile peaked on axis is incident into a plasma, self-focusing and selfchanneling can occur as a result of two effects: the relativistic modification of electron mass in the laser field $[\gamma(0)>\gamma(r)]$ and the reduction of the electron density on axis due to the expulsion of electrons by laser ponderomotive force $\left[n_{e}(0)<n_{e}(r)\right]$. It was predicted $[5,6]$ that such self-channeling should occur-even in the absence of ion motion-provided the laser power exceeds a criti- cal power given by $P_{c}=17\left(\omega_{0} / \omega_{p}\right)^{2} \mathrm{GW}$. Two groups have already demonstrated relativistic-ponderomotive selfchanneling experimentally $[7,8]$ by means of side imaging of Thomson scattering [9].

In ponderomotive self-channeling, the laser ponderomotive force expels electrons from the axis (the ions do not move much because of their greater mass) and prevents their return, despite the Coulomb force, which arises from charge separation. If the laser pulse duration is long enough, the ions can start to move out as a result of this Coulomb force and gain momenta during the process. After the laser pulse is gone, electrons quickly return in order to neutralize the bare ions. However, the ions (and electrons) keep moving out of the axis as a result of the ion momentum gained during the laser pulse. This is predicted to lead to the formation of a plasma-density depression on axis $[10,11]$, which becomes deeper and wider with time. Such a plasma-density depression forms a channel (plasma waveguide) which can be used to guide a second laser pulse. A plasma waveguide preformed in this way has advantages: a narrow channel width $(\leq 30 \mu \mathrm{m})$, high-plasma density $\left(\geq 10^{19} \mathrm{~cm}^{-3}\right)$ and the ability to sustain high-laser intensity $\left(\geq 10^{18} \mathrm{~W} / \mathrm{cm}^{2}\right)$, as compared with waveguides formed by long-duration laser pulses via thermal hydrodynamic expansion followed by shock-front formation [12].

Several groups $[13,14]$ have claimed to observe plasma-density-depression channels produced in this way. However, these experiments did not present quantitative measurement of the evolution of a plasma waveguide and so other underlying mechanisms, such as thermal shock blowout after anomalously strong laser heating, were not experimentally ruled out. Although such a plasma waveguide has also been investigated by Krushelnick et al. [15], its existence was supported only indirectly by the guiding of a trailing laser pulse and no measurements of the density depression were made. In this Letter, we report the direct experimental observation of a plasma waveguide created from laser ponderomotive self-channeling in a uniform plasma and characterize the formation of such a plasma waveguide. Probing interferometry provided detailed time- and space-resolved information on the 
evolution of the plasma-density profile. A trailing pulse that is collinear with the channel-forming pulse is used to probe the guiding capability and guiding modes of the plasma waveguide. The observed guiding is well correlated with the interferometric results and, in addition, reveals the effect of ponderomotive self-channeling during the pump (channel-making) pulse.

In this experiment, we used a Ti:sapphire-Nd:glass laser system based on chirped-pulse amplification that produces $2 \mathrm{~J}, 400$-fs pulses at $1.053 \mu \mathrm{m}$. The $50 \mathrm{~mm}$ diameter laser pulse was focused with an $f / 3.3$-off-axis parabolic mirror to $6.7 \mu \mathrm{m}$ FWHM, corresponding to vacuum intensity exceeding $6 \times 10^{18} \mathrm{~W} / \mathrm{cm}^{2}$. This pulse was focused onto a supersonic helium gas jet with a sharp gradient $(250 \mu \mathrm{m})$ and a long flat-topped interaction region $(1 \mathrm{~mm})$. At the maximum gas jet backing pressure, an underdense plasma with $4 \times 10^{19}-\mathrm{cm}^{-3}$ plasma density is formed by the foot of the laser pulse tunnel ionizing the gas. This plasma density corresponds to a critical power of $P_{c}=430 \mathrm{GW}$.

In order to diagnose the spatial extent of laser channel, both side imaging of Thomson scattering and near-field imaging of the channel exit are used simultaneously. From side imaging of Thomson scattering, it is observed that the length of laser channel increases stepwise with the increase of laser power [8]. Below 1.5 TW, the laser channel extends longer with increasing power from 200 to $400 \mu \mathrm{m}$ for a fixed plasma density of $4 \times 10^{19} \mathrm{~cm}^{-3}$. The channel length extends abruptly to $700 \mu \mathrm{m}$ when the laser power exceeds $1.5 \mathrm{TW}\left(3.5 P_{c}\right)$ and extends again to $1 \mathrm{~mm}\left(10 Z_{R}\right.$, limited by the gas jet length) for $2 \mathrm{TW}\left(4.6 P_{c}\right)$ and above. When the laser power is fixed and gas density is varied, similar behavior in the channel length is observed except that the change in length is more gradual. The channel diameter is measured to be less than $12 \mu \mathrm{m}$ along the entire 1-mm channel, limited by the resolution of side imaging of Thomson scattering.

When the peak power of the laser pulse exceeds the guiding threshold (including the effects of ionization defocusing), not all of the energy in the pulse is guided. Only the temporal central part of the pulse, in which the power exceeds the critical power threshold, is guided, while the rest erodes away due to divergence. In addition, if the transverse profile is not perfectly adapted to the ideal stable beam profile, the nonideal part (e.g., wings) will also not be guided. Imaging of the laser beam profile near the focus is accomplished by image relaying the beam to a microscope objective, which images it onto a charge-coupled device camera. If the imaging plane of this near-field imaging system is located at the far (exit) end of the gas jet, the transverse profile of the laser beam at this position changes significantly when self-channeling occurs. Figure 1 shows the change in laser beam profile at this position as a function of laser power and gas density. At laser power below $1.5 \mathrm{TW}$, the laser beam breaks up and the beam profile at this position is large $(100 \mu \mathrm{m})$. When laser power exceeds $1.5 \mathrm{TW}$, part of the laser energy starts to form a channel. As the laser channel extends to this imaging plane

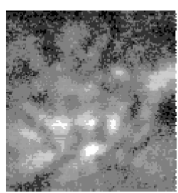

(a)

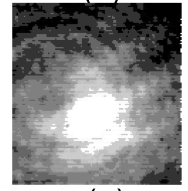

(e)

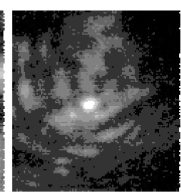

(b)

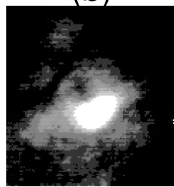

(f)

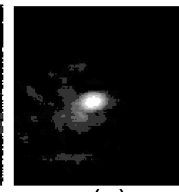

(c)

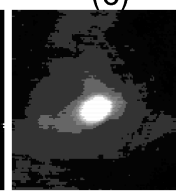

(g)

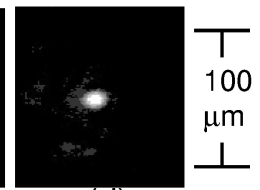

(d)

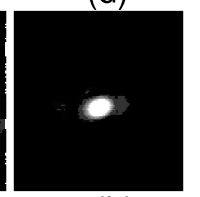

(h)

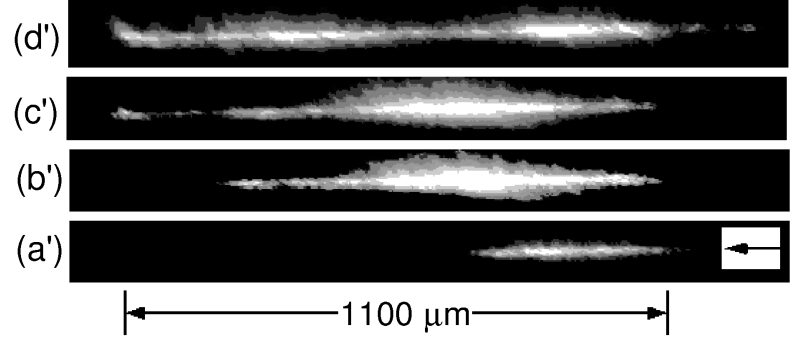

FIG. 1. Images of laser beam profiles at the end of gas jet. (a) -(d) are at various laser powers and a fixed electron density of $4 \times 10^{19} \mathrm{~cm}^{-3}$ : (a) $1.0 \mathrm{TW}$, (b) $1.5 \mathrm{TW}$, (c) $2.0 \mathrm{TW}$, and (d) $2.5 \mathrm{TW}$. (e) $-(\mathrm{h})$ and (d) are at various gas density and a fixed laser power of $2.5 \mathrm{TW}$ : (e) $2.2 \times 10^{18}$, (f) $6.7 \times 10^{18}$, (g) $1.1 \times 10^{19}$, (h) $1.6 \times 10^{19}$, and (d) $2.0 \times 10^{19} \mathrm{~cm}^{-3}$. (a')$\left(d^{\prime}\right)$ show the side imaging of laser beams corresponding to (a)-(d), respectively. Arrow inset indicates the direction of laser propagation.

at $2 \mathrm{TW}$ and above, the laser beam profile is observed to consist of a round spot which is smooth and small in size (10 $\mu \mathrm{m}$, limited by the resolution of the imaging relay system), plus a large dim spot accounting for the unguided part. When the laser power is fixed at $2.5 \mathrm{TW}$ and the gas density is varied, it is observed that the laser pulse always focuses to a smooth Gaussian spot. In addition, both the decrease in the size of the laser beam profile and the increase in the channel length with increasing gas density are found to be gradual. These observations are consistent with the variation of the channel length and diameter with laser power and gas density observed with the side imaging of Thomson scattering. We measured that about $45 \%$ of the pulse energy is guided at high powers.

In order to observe the formation of the plasma waveguide, probing interferometry was used to obtain images of the plasma density distribution at different times. A probe pulse $(400 \mathrm{fs}, 1.053 \mu \mathrm{m})$ is obtained by splitting $5 \%$ of the pump pulse, sending it into a delay line, and crossing it perpendicularly with the pump pulse in the interaction region. Interferograms were obtained by use of two glass wedges and imaging optics [16]. Spatial resolution is about $3 \mu \mathrm{m}$ in the radial direction and $50 \mu \mathrm{m}$ in the longitudinal direction (the fringe separation). Temporal resolution is determined by the probe pulse duration, about 400 fs. Figure 2 shows interferograms for a 2.5 -TW pump pulse and gas density of $2 \times 10^{19} \mathrm{~cm}^{-3}$ at delays of 5 and 30 ps. A nearly $100 \%$ density depression on axis is 


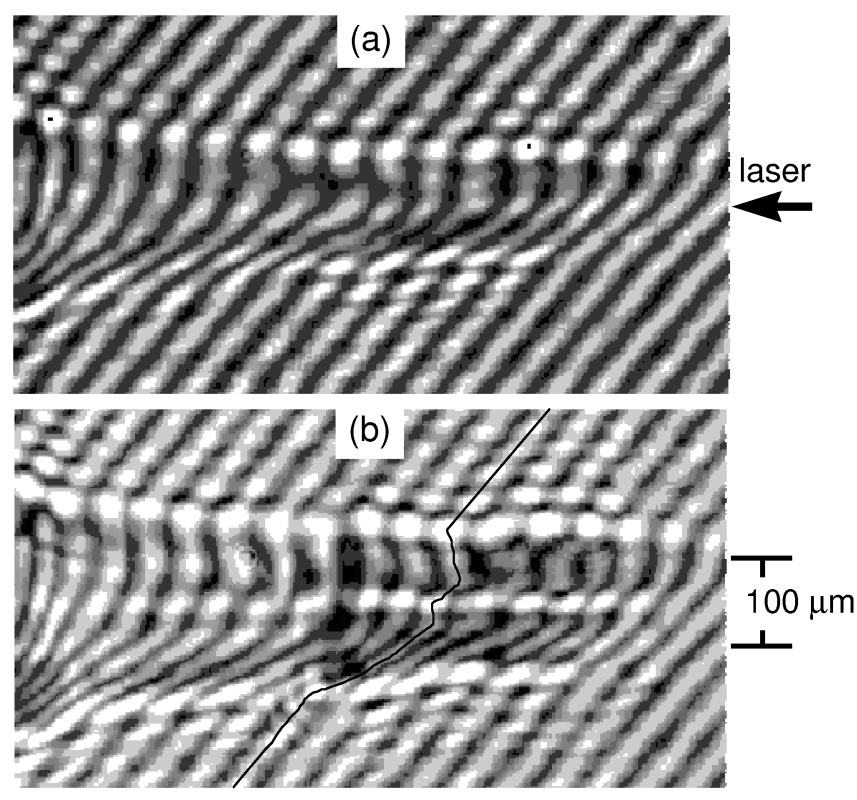

FIG. 2. Interferograms of the plasma taken at different times for 2.5-TW laser power and $2 \times 10^{19}-\mathrm{cm}^{-3}$ gas density: (a) 5 ps and (b) 30 ps. (b) shows the development of a density depression on axis. The solid line is to help view the fringes.

observed $25 \mathrm{ps}$ after the passage of the pump pulse. Under some circumstances (e.g., higher gas density), clear density depression channel is observed in the interferograms as early as 2 ps after the pump pulse. By varying the laser power and gas density at a fixed delay (25 ps), we observed that the plasma waveguide overlaps with the pump-pulse laser channel seen from side imaging of Thomson scattering and extends in length accordingly as the laser channel of the pump pulse extends. This is consistent with the conclusion that ponderomotive self-channeling of the laser is the source for plasma waveguide formation in this experiment.

To determine quantitatively the evolution of the plasma waveguide, a 3D plasma density distribution was obtained by means of a fringe tracking program (to get a 2D phaseshift distribution) in conjunction with an Abel inversion algorithm. Figure 3 shows the 3D plasma density distribution (assuming cylindrical symmetry) evolving in time. As can be seen, the density depression on axis becomes deeper and the width of the channel becomes larger as time goes by. At about $40 \mathrm{ps}$ delay, a plasma waveguide of $900 \mu \mathrm{m}$ in length is formed, which has an on-axis plasma density less than $10^{18} \mathrm{~cm}^{-3}$ and a channel width of $30 \mu \mathrm{m}$. The velocity of ions after the passage of the laser pulse is roughly given by $\left\langle Z m_{e} c^{2} \int d t\left(\nabla_{r} \sqrt{1+a^{2} / 2}\right) / m_{i}\right\rangle \simeq$ $Z m_{e} c^{2} / m_{i} \cdot\left(\sqrt{1+a_{0}^{2} / 2}-1\right) \tau / r_{0}$, where $Z$ is the ion charge, $\tau$ is the pulse duration, and $r_{0}$ is the radius of the laser channel. At $2.5 \mathrm{TW}$ laser power (and $45 \%$ guiding), the mean ion velocity is about $1 \mu \mathrm{m} / \mathrm{ps}$. This is consistent with the measured speed of waveguide formation $(\sim 1 \mu \mathrm{m} / \mathrm{ps})$. Figure 3 also shows the increase of the diameter of plasma region with time at a speed of approxi-
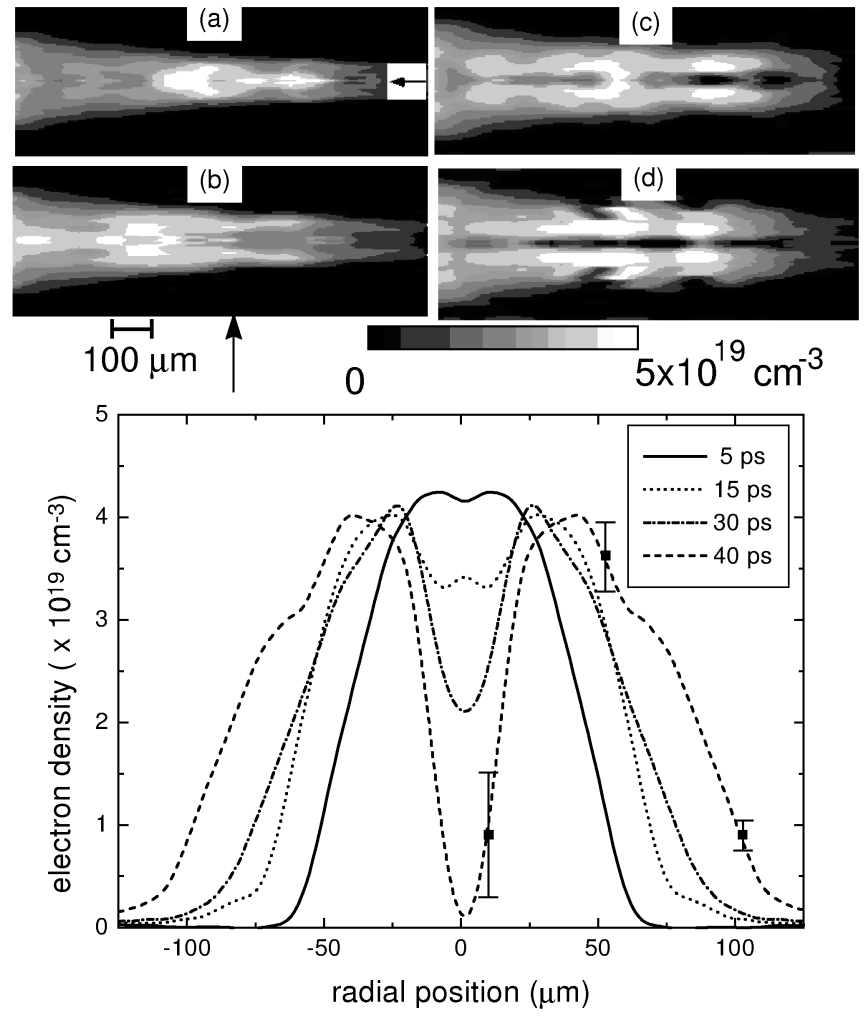

FIG. 3. 3D plasma density distribution for 2.5-TW laser power and $2 \times 10^{19}-\mathrm{cm}^{-3}$ gas density at different times:

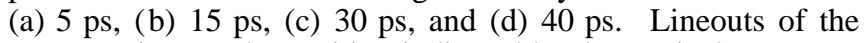
cross sections at the position indicated by the vertical arrow are shown along with the error bars at three selected positions.

mately $1 \mu \mathrm{m} / \mathrm{ps}$ (about 10 times the thermal expansion velocity at $100 \mathrm{eV}$ temperature). This expansion is due to production of new plasma in the radial direction caused by collisional ionization. The size of the initial plasma region is determined by ionization by the unguided part (55\% at high powers) of the laser beam, which is defocused to about $100 \mu \mathrm{m}$ FWHM. This gives a laser intensity of $1.5 \times 10^{16} \mathrm{~W} / \mathrm{cm}^{2}$, enough to ionize the entire $100-\mu \mathrm{m}$-diam plasma region.

To observe the guiding capability of the electron density depression and the subsequent plasma waveguide, a probe pulse (400-fs duration, 527-nm wavelength, 200$\mathrm{mJ}$ maximum energy) generated from the pump pulse is propagated collinearly with the pump pulse. The imaging system for measuring the pump beam profile was modified to study the guiding of the probe pulse. For $2.5-\mathrm{TW}$ peak laser power and $4 \times 10^{19}-\mathrm{cm}^{-3}$ plasma density, the beam profile of the probe pulse at the exit of the plasma waveguide changes from 100 to $7 \mu \mathrm{m}$ in diameter, as the probe delay changes from negative to positive (i.e., prepulse to postpulse). By scanning the imaging plane at different delays, we found out that the guided length of probe pulse increases with probe delay. The guiding length increases from $600 \mu \mathrm{m}$ at zero delay to $1100 \mu \mathrm{m}$ at $1.5 \mathrm{ps}$ delay and maintains this length for longer delays (see Fig. 4). The same results are also observed in the side imaging of 
(a)

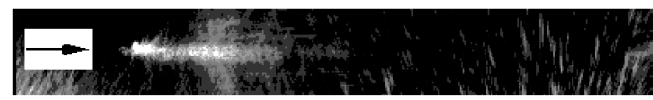

(b)

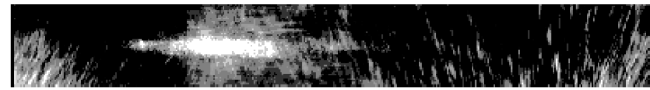

(c)

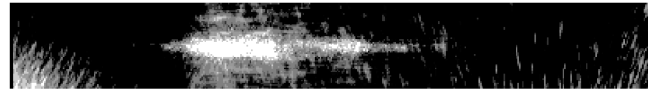

(d)
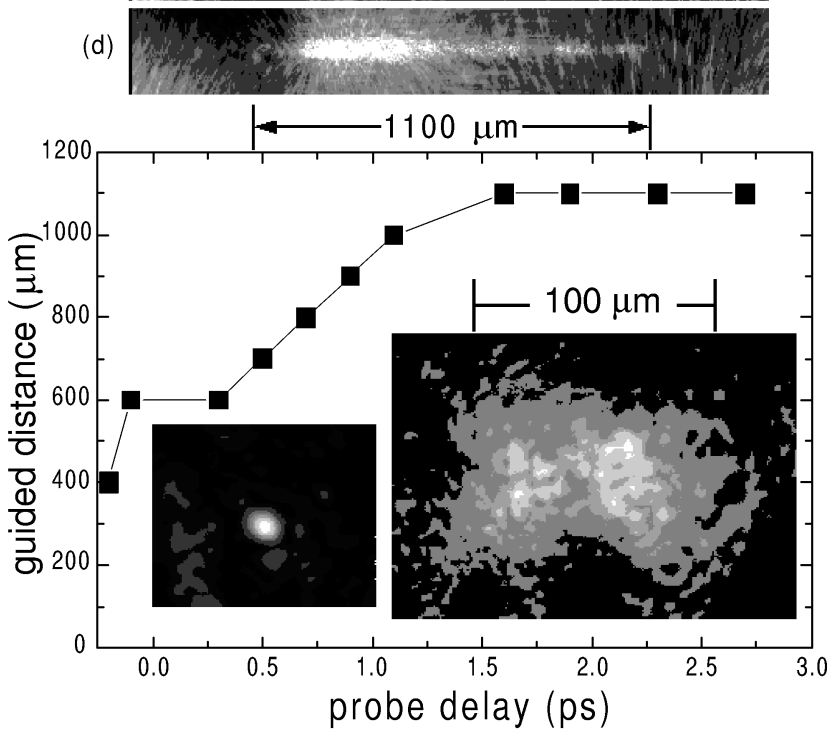

FIG. 4. Guiding length of the probe pulse at early delays. The ultimate length is limited by the gas jet. Insets are the laser beam profile at the end of the gas jet with 2-ps delay (left) and without the pump (right). (a)-(d) shows the side imaging of the probe at different delays: (a) $-200 \mathrm{fs}$, (b) $+300 \mathrm{fs}$, (c) $+900 \mathrm{fs},(\mathrm{d})+2.7 \mathrm{ps}$.

Thomson scattering of the probe (Fig. 4). When the probe delay is longer than $20 \mathrm{ps}$, the width of the waveguide is large enough $(30 \mu \mathrm{m})$ to accommodate higher order guiding modes and, thus, multiple-spot modes were observed with a diameter of $\sim 30 \mu \mathrm{m}$. The probe guiding results indicate that a plasma waveguide capable of guiding an intense laser pulse is formed during and after the passage of a ponderomotively self-channeled laser pulse. This is not observed clearly in the interferometric result because the phase shift from this small density depression $(\sim 10 \%)$ and small channel width $(\sim 10 \mu \mathrm{m})$ is less than the errors from the phase front distortion of the probe beam and the Abel inversion procedure. A simple estimate [4] indicates that a parabolic density channel will guide a Gaussian laser beam provided that the depth of the density channel is larger than $\Delta n=1 / \pi r_{e} r_{L}^{2}$, where $\Delta n=n_{e}\left(r_{L}\right)-n_{e}(0)$, $r_{L}$ is the channel radius, and $r_{e}$ is the classical electron radius. This requires a density depression larger than $11 \%$ for a $10-\mu \mathrm{m}$ channel. By integrating the signals in the images of guided beam profile at the channel exit, it is found that $65 \% \pm 10 \%$ of probe energy is guided.

Although the guiding of a trailing pulse can also be caused by the temporary waveguide formed by the relativistic modification of the refractive index (at -200 - to
+200 -fs probe delay), due to the pump pulse, or by the plasma wave (at $\sim-1$ - to +2 -ps probe delay) excited by the pump pulse, the gradual development of guiding channel observed from zero delay to over tens of picoseconds indicates that the plasma density depression is the dominant guiding force even at early delay times. The ability of this plasma waveguide to guide the probe pulse at zero delay proves the existence and effect of relativisticponderomotive self-channeling on the pump pulse itself. The formation of the plasma waveguide is believed to be driven by ion momentum following the ponderomotive self-channeling of pump pulse. Another possibility for the formation of the on-axis plasma-density depression may come from the high-temperature heating on axis due to wave breaking of plasma wave excited by Raman backscattering and sidescattering. However, this driving force, $\nabla\left(k T_{e}\right)$, is negligible compared to the ponderomotive driving force, $\nabla\left(\Phi_{p}\right)$, because the electron temperature $k T_{e}\left(\simeq m_{e} v_{p}^{2} \simeq 10 \mathrm{keV}\right.$, where $v_{p}$ is the phase velocity of the plasma wave) is much smaller than the ponderomotive potential $\Phi_{p}(\simeq 300 \mathrm{keV})$, and the diameter of plasma wave and the laser focus are roughly the same. It is therefore believed that the mechanism of ponderomotive selfchanneling is dominant for this work and probably for that of [13-15] as well. The observation of an intense laser pulse $\left(3 \times 10^{17} \mathrm{~W} / \mathrm{cm}^{2}\right)$ guided in a high density waveguide ( $\geq 10^{19} \mathrm{~cm}^{-3}$ on axis) is relevant to several important practical applications.

This work was supported by NSF PHY 972661 and NSF STC PHY 8920108. We also acknowledge the valuable contributions of M. Krishnan, G. Mourou, and K. Teo.

[1] T. Tajima and J. Dawson, Phys. Rev. Lett. 43, 267 (1979).

[2] D. Umstadter et al., Science 273, 472 (1996).

[3] M. Tabak et al., Phys. Plasmas 1, 1626 (1994).

[4] E. Esarey et al., IEEE Trans. Plasma Sci. 24, 252 (1996); IEEE J. Quantum Electron. 33, 1879 (1997).

[5] G. Z. Sun et al., Phys. Fluids 30, 526 (1987).

[6] A. B. Borisov et al., Phys. Rev. A 45, 5830 (1992).

[7] P. Monot et al., Phys. Rev. Lett. 74, 2953 (1995).

[8] R. Wagner et al., Phys. Rev. Lett. 78, 3125 (1997).

[9] A. Sullivan et al., Opt. Lett. 19, 1544 (1994).

[10] D. C. Barnes, T. Kurki-Suonio, and T. Tajima, IEEE Trans. Plasma Sci. 15, 154 (1987).

[11] A. Pukhov and J. Meyer-ter-Vehn, Phys. Rev. Lett. 76, 3975 (1996).

[12] L. C. Johnson and T. K. Chu, Phys. Rev. Lett. 32, 517 (1974); T.R. Clark et al., Phys. Rev. Lett. 78, 2373 (1997); V. Malka et al., Phys. Rev. Lett. 79, 2979 (1997).

[13] M. Borghesi et al., Phys. Rev. Lett. 78, 879 (1997).

[14] G. Malka et al., Phys. Rev. Lett. 79, 2053 (1997).

[15] K. Krushelnick et al., Phys. Rev. Lett. 78, 4047 (1997).

[16] G. S. Sarkisov, Instrum. Exp. Tech. 39, 727 (1996). 